\title{
A New Surgical Model of Skeletal Muscle Injuries in Rats Reproduces Human Sports Lesions
}

Authors

Affiliations
P. Contreras-Muñoz ${ }^{1,2}$, A. Fernández-Martín ${ }^{2}$, R. Torrella ${ }^{3}$, X. Serres ${ }^{4}$, M. De la Varga ${ }^{1}$, G. Viscor ${ }^{3}$, T. A. H. Järvinen ${ }^{5,6}$, V. Martínez-lbáñez ${ }^{2}$, J. L. Peiró ${ }^{2,7}$, G. Rodas $^{1,8}$, M. Marotta ${ }^{1,2}$

Affiliation addresses are listed at the end of the article

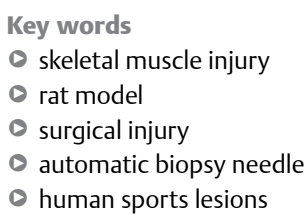

accepted after revision June 18, 2015

\section{Bibliography}

DOI http://dx.doi.org/ 10.1055/s-0035-1555933

Published online:

December 15, 2015

Int J Sports Med 2016; 37:

183-190 @ Georg Thieme Verlag KG Stuttgart . New York ISSN 0172-4622

\section{Correspondence}

\section{Dr. Mario Marotta}

Leitat Foundation MuscleTech Network C/Innovació no 2 08225 Terrassa (Barcelona) Spain

Tel.: + 34934893000

Ext.: 3789

Fax: + 34934893893

mmarotta@fundacionleitat.org

\section{Abstract}

$\nabla$

Skeletal muscle injuries are the most common sports-related injuries in sports medicine. In this work, we have generated a new surgicallyinduced skeletal muscle injury in rats, by using a biopsy needle, which could be easily reproduced and highly mimics skeletal muscle lesions detected in human athletes. By means of histology, immunofluorescence and MRI imaging, we corroborated that our model reproduced the necrosis, inflammation and regeneration processes observed in dystrophic mdx-mice, a model of spontaneous muscle injury, and realistically mimicked the muscle lesions observed in professional athletes. Surgically-injured rat skeletal muscles demonstrated the longitudinal process

\section{Introduction}

$\nabla$

Skeletal muscle injuries are the most common sports-related injuries (their frequency varying from $10-55 \%$ of all sustained injuries) and a major concern in sports medicine $[2,19]$. According to the World Health Organization (WHO), musculoskeletal injuries are the most common cause of severe long-term pain and physical disability, and affect hundreds of millions of people worldwide [38]. Loss or abnormality of bodily structure or functioning resulting from an isolated exposure to physical energy during sports training or competition are normally diagnosed by a clinical professional as a medically recognized injury [33].

In human sport clinics, the diagnosis of muscle injury is adequate in most cases, but imaging modalities such as ultrasonography, computed tomography (CT) or magnetic resonance imaging (MRI) are important to differentiate between structural lesions and functional disorders and to determine the extent of the injury although of muscle regeneration and fibrogenesis as stated by Myosin Heavy Chain developmental (MHCd) and collagen-I protein expression. MRI imaging analysis demonstrated that our muscle injury model reproduces the grade I-II type lesions detected in professional soccer players, including edema around the central tendon and the typically high signal feather shape along muscle fibers. A significant reduction of $30 \%$ in maximum tetanus force was also registered after 2 weeks of muscle injury. This new model represents an excellent approach to the study of the mechanisms of muscle injury and repair, and could open new avenues for developing innovative therapeutic approaches to skeletal muscle regeneration in sports medicine.

small and deep hematomas localized within the muscle belly could be more difficult to diagnose clinically $[1,23,32]$. Ultrasonography can be considered a valid first-line tool, if a more exact characterization of the injury is desired. MRI, in turn, should be preferred if a clear discrepancy exists between the patient's symptoms, the physician's findings and/or the ultrasonography and, in particular, in injuries close to the muscle-tendon junction [1,32], where MRI has shown its superiority over ultrasonography [18]. MRI can accurately confirm or rule out the existence of muscle injury and also provides a very detailed characterization of the muscle lesion [18]. At present, there is much controversy in the classification of skeletal muscle injuries, which sometimes causes limitations to the comprehensive study of muscle injuries and discrepancies in the uniformity for their categorization and description [10]. Based in the last classification proposed by Pollock et al. [27], skeletal muscle lesions could be classified in 4 grades depending on MRI imaging: a) Grade I: These are small injuries of the 
muscle characterized by high signal change (edema in T2), which is evident at this site and extends over a limited area of less than $5 \mathrm{~cm}$ and less than $10 \%$ of the muscle cross-sectional area at its maximal site; b) Grade II: These types of injuries are moderate injuries of the muscle. Under MRI imaging, the high signal change will either measure between 10 and $50 \%$ of the crosssectional area of that individual muscle at the site of injury or extend between 5 and $15 \mathrm{~cm}$ within the muscle. Architectural fiber disruption is commonly less than $5 \mathrm{~cm}$; c) Grade III: These injuries are extensive injuries of the muscle. MRI features of high signal change patterns of greater than $50 \%$ of the muscle crosssectional area or greater than $15 \mathrm{~cm}$ in length. It is evidenced that the architectural muscle fiber disruption which is likely to be greater than $5 \mathrm{~cm}$; d) Grade IV: These are the most severe injuries and are characterized by a complete tear of either the muscle or tendon.

Skeletal muscle lesions are often caused by eccentric contraction that forcibly lengthens an activated muscle. When unaccustomed or intense, this active stretching of the muscle-tendon unit can produce exercise-induced muscle injury [7]. Clinical data reveal a high recurrence rate (nearly $30 \%$ ) of skeletal muscle strain injuries among professional athletes [37], and a main concern in professional athletes is the faster recovery after trauma [4]. The consequences of failed treatment can be very dramatic, possibly postponing an athlete's return to play for weeks or even months. Thus the recognition of some basic principles of skeletal muscle regeneration and healing processes can considerably help in both avoiding the imminent dangers and accelerating the return to competition [17]

Adult skeletal muscle has the ability to self-repair in response to injury $[6,13]$ and commonly the muscle tissue is damaged and repaired repeatedly throughout life, especially in sportsmen. The natural history of muscle injury proceeds through a highly coordinated sequence of steps. Muscle regeneration is a highly coordinated process that requires the utilization of undifferentiated cells, called satellite cells (or myogenic progenitor cells) located between the basal lamina and the sarcolemma of mature muscle fibers $[3,9,13,31]$. Satellite cell population is a residual pool of self-renewing progenitor cells, capable of supporting additional rounds of regeneration and to reestablish the initial quiescent satellite cell's pool after every muscle injury episode [8]. When activated by muscle damage, they can proliferate producing a large number of myoblasts that can repair skeletal muscle injury and generate a large number of new myofibers within just a few days [36]. Myoblasts can fuse and differentiate into multinucleated fibers recapitulating the events of embryonic muscle development and finally restoring the skeletal muscle architecture $[6,13,25,28,31]$. The complex muscle repair process is dependent on an orchestrated response between the inductive signals of cytokines and growth factors, which are released by the injured fibers, invading macrophages and inflammatory cells or connective tissue $[6,9]$. Simultaneously, increased expression of angiogenic factors and markers of new capillaries, such as vascular endothelial growth factor (VEGF), angiopoeitin- $1 / 2$ and $\alpha$-smooth muscle actin ( $\alpha$-SMA), could be detected along with the formation of new myofibers ensuring the maintenance of blood supply to regenerated muscle $[30,35]$. The repair of skeletal muscle follows a set pattern, which can be divided into 3 phases [15,16,18,19,21]: 1) Destruction phase, characterized by the rupture of the membrane of muscle fibers and the subsequent necrosis and inflammatory cell reaction; 2) Repair phase, characterized by the phagocytosis of the necrotic muscle fibers by blood derived monocytes, satellite cells activation, generation of new muscle fibers and the production of a tissue scar; 3 ) Remodeling phase, characterized by the reorganization of the muscle fibers, the remodeling of the scar tissue, and the restoration of muscle function and maturation of the regenerating myofibers including the formation of a mature contractile apparatus. Even though in the last 25 years extensive research has been directed towards the elucidation of the process of skeletal muscle regeneration, its complex regulatory pathways still remain poorly understood [22].

In this study, we present a new in vivo model of surgicallyinduced lesions that could mimic the most frequent skeletal muscle lesions observed in the human sports clinics. Hence, in the present work our aim was to generate a new surgicallyinduced skeletal muscle injury model in rats that could be easily reproduced and that mimicked the skeletal muscle lesions observed in human elite athletes and sportsmen which could open new avenues for future studies of innovative therapeutic approaches to skeletal muscle regeneration and sports medicine.

\section{Materials and Methods}

$\nabla$

\section{Animals}

Adult (8-week-old) male Wistar rats (Harlan Laboratories, Indianapolis, IN) were used as animal model. The total number of animals used in our study was 63 . Rats were housed at $22-24^{\circ} \mathrm{C}$ and maintained on a 12-h light/dark cycle. Water and food were given ad libitum during the experiments. All procedures were performed in accordance with Spanish (Real Decreto 53/2013) and European (2010/63/UE) legislation and approved by the Departament d'Agricultura, Ramaderia, Pesca, Alimentació i Medi Natural of the Catalan Government (Generalitat de Catalunya).

\section{Surgically-induced skeletal muscle injury rat model}

Rats were anaesthetized by an intraperitoneal injection of a mixture of ketamine ( $75 \mathrm{mg} / \mathrm{kg}$, i.p.) and xylazine $(10 \mathrm{mg} / \mathrm{kg}$, i.p.) before surgical procedure and placed in prone position by the fixation of tail and extremities with adhesive strips to a cork surface exposing the ventral side of the right crus. The surgical procedure for creating skeletal traumatic muscle lesion in the rat gastrocnemius muscle is illustrated in $\bullet$ Fig. 1. The muscle injury was generated by using an $18 \mathrm{~g}$ biopsy needle $\left(\operatorname{Bard}^{\circledR}\right.$ Monopty ${ }^{\circledR}$ Disposable Core Biopsy Instrument, Bard Biopsy Systems, Tempe, USA) with $0.84 \mathrm{~mm}$ inner diameter. Transversal biopsy procedure was performed at the myotendinous junction level of the right leg medial gastrocnemius muscle $(3 \mathrm{~mm}$ from the start of muscle-tendon junction and $2 \mathrm{~mm}$ in depth) after shaving the lower extremity and cleaning with ethanol to identify the white Achilles tendon and measure the injury site from the muscletendon junction site. Post-surgical analgesia (buprenorphine $0.01 \mathrm{mg} / \mathrm{kg}$ ) was subcutaneously administered to all operated animals. Our study conforms to the ethical standards in sport and exercise science research [12].

\section{Measurement of muscle injury size in rat medial gastrocnemius muscle}

The size of the muscle lesion caused by the biopsy needle in the medial gastrocnemius muscle was measured by measuring the areas in Hematoxylin-Eosin histology images from rat gastrocnemius muscle sections ( $N=5$ for each time-point) at $1,3,5,7$, $14,21,26,35$ and 46 days post-injury. Microphotographs were 


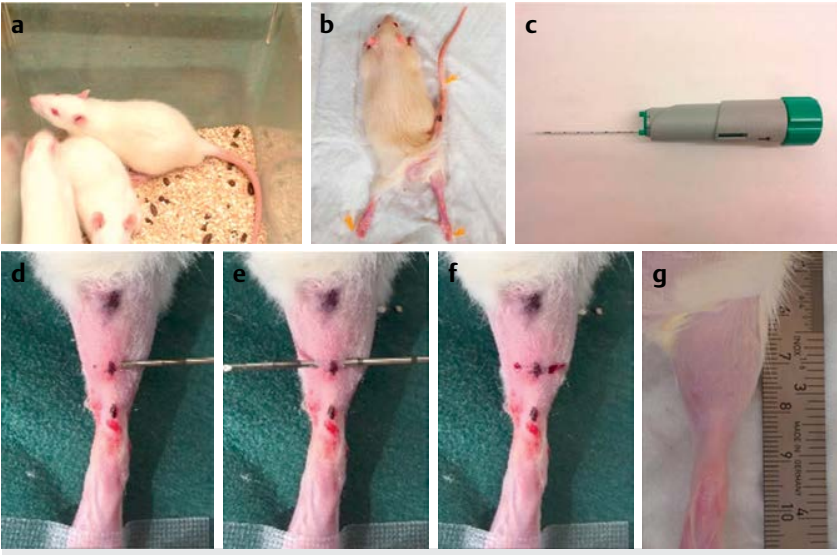

Fig. 1 Surgically-induced skeletal muscle injury rat model. a Male Wistar rats were used as the animal model for muscle injury induction using the automatic biopsy needle. $\mathbf{b}$ Animals were anaesthetized before surgical procedure and placed in prone position by the fixation of tail and extremities. c Skeletal muscle injury was generated by using an $18 \mathrm{~g}$ biopsy needle (Bard ${ }^{\circledR}$ Monopty ${ }^{\circledR}$ Disposable Core Biopsy Instrument, Bard Biopsy Systems, Tempe, USA). d Transverse biopsy procedure in medial gastrocnemius muscle was generated by using the biopsy needle at $3 \mathrm{~mm}$ proximal from the muscle-tendon junction site. e The lesion spans roughly $50 \%$ of the cross-sectional area of the medial gastrocnemius muscle. f After biopsy, needle was extracted and external antiseptic was applied. Post-surgical analgesia was subcutaneously administered to all operated animals. $g$ One week after surgery the puncture wounds created from the entrance and exit of the biopsy needle were closed.

obtained by using a BX-61 microscope (Olympus) equipped with a DP72 camera (Olympus) and the CellSens ${ }^{\circledR}$ Digital Imaging software (version 1.9). The areas of the injured muscle region and the total medial gastrocnemius were quantified by using the Image J v1.46 software. The size of the muscle lesion was represented by the percentage of the injured area vs. the total area of the medial gastrocnemius muscle.

\section{Histology and immunofluorescence analysis}

Animals ( $\mathrm{N}=5$ for each time point) were euthanized by intraperitoneal injection of an overdose of ketamine $(75 \mathrm{mg} / \mathrm{kg}$ ) and xylazine $(10 \mathrm{mg} / \mathrm{kg})$ at $1,3,5,7,10,12,14,18,21$ and 26 days after the injury. Gastrocnemius muscles were excised, and immediately frozen in 2-methylbutane (Alfa Aesar. A Johnson Matthey Company, Karlsruhe, Germany), which was previously super-cooled in liquid nitrogen, and stored at $-80^{\circ} \mathrm{C}$ until analysis. Frozen medial gastrocnemius were transversely sectioned (10 $\mu \mathrm{m}$ thick) using a Cryotome (Leica Microsystems, Wetzlar, Germany) at below $-20^{\circ} \mathrm{C}$ and mounted on Polylisine ${ }^{\mathrm{TM}}$ glass slides (VWR, Leuven, Belgium). Consecutive frozen muscle sections were used for histological and immunofluorescence analysis. For histological analysis, rat skeletal muscle sections were stained with Hematoxylin-Eosin (1 min hematoxylin and $15 \mathrm{~s}$ eosin), washed in water, dehydrated with graded ethanol solutions ( 1 min ethanol 50\%, 1 min ethanol 70\% twice, 1 min ethanol $90 \%, 1$ min ethanol 100\% twice) and cleared in xylene (5s). After air-drying for $5 \mathrm{~min}$, the slides were mounted with DPX mounting medium and a coverslip (VWR, Madrid, Spain). Hematoxylin Harris solution was purchased from Casa Álvarez (Casa Álvarez, Madrid, España) and eosin solution was prepared by dissolving 0.5 g Eosin Yellowish (Panreac Quimica SA, Barcelona, Spain) in $100 \mathrm{ml}$ water with $200 \mu \mathrm{l}$ glacial acetic acid (Sigma-
Aldrich Quimica SA, Madrid, Spain). Ethanol absolute and xylene were obtained from VWR (VWR, Leuven, Belgium).

For immunofluorescence analysis, frozen muscle sections were fixed in cold $\left(-20^{\circ} \mathrm{C}\right.$ ) acetone (Sigma-Aldrich Quimica SA, Madrid, Spain) for $5 \mathrm{~min}$, air-dried and blocked in PBS containing 3\% BSA (bovine serum albumin, Sigma-Aldrich Quimica SA, Madrid, Spain) for $10 \mathrm{~min}$ at room temperature and then incubated with primary antibodies diluted 1:100 in PBS (Phosphate buffered saline, Biowest, Barcelona, Spain). The primary antibodies used were against rabbit anti-Collagen I (Abcam, Cambridge, UK), mouse $\alpha$-smooth muscle actin ( $\alpha$-SMA) (SigmaAldrich Quimica SA, Madrid, Spain) and mouse developmental Myosin Heavy Chain (MHCd, Novocastra, Newcastle, UK) for $16 \mathrm{~h}$ in a humid chamber at $4^{\circ} \mathrm{C}$. Samples were washed 3 times in PBS and incubated with the secondary antibodies Alexa Fluor ${ }^{\circledR} 568$ anti-mouse or Alexa Fluor ${ }^{\circledR} 488$ anti-rabbit (Invitrogen, Oregon, USA) diluted 1:1000 in PBS in a dark humid chamber for $1 \mathrm{~h}$ at room temperature. Finally, slides were washed 3 times with PBS and mounted using a coverslip and Fluoromount-G (Southern Biotech, Madrid, Spain) mounting medium. Fluorescence was evaluated by using a BX-61 microscope (Olympus) using a DP72 camera (Olympus) and CellSens ${ }^{\circledR}$ Digital Imaging software (version 1.9).

\section{Imaging studies by Magnetic Resonance (MRI)}

In vivo ${ }^{1} \mathrm{H}$-magnetic resonance imaging (MRI) studies were performed daily during 4 weeks at the NMR facilities of the Autonomous University of Barcelona (UAB, Barcelona, Spain) using a $7 \mathrm{~T}$ Bruker BioSpec 70/30 USR (Bruker BioSpin GmbH, Ettlingen, Germany) system equipped with a mini-imaging gradient set $(400 \mathrm{mT} / \mathrm{m})$ and using a quadrature transceiver volume coil with $72 \mathrm{~mm}$ inner diameter.

Rats $(\mathrm{N}=3)$ were positioned in a bed, which allowed delivery of anesthesia (isoflurane, $1.5-2.0 \%$ in $\mathrm{O}_{2}$ at $1 \mathrm{~L} / \mathrm{min}$ ), with an integrated heat water circuit for body temperature regulation. Body temperature was measured with a rectal probe and maintained at $37 \pm 1^{\circ} \mathrm{C}$. Respiratory frequency was monitored with a pressure probe and kept between $60-80$ breaths/min. Low resolution T2-weighted fast spin-echo images were initially obtained in axial, sagittal and coronal planes to be used as reference scout images. Imaging parameters for these images were: effective echo time $($ TEeff $)=36 \mathrm{~ms}$; repetition time $(T R)=3 \mathrm{~s}$; echo train length $(\mathrm{ETL})=8$; field of view $(\mathrm{FOV})=6 \times 6 \mathrm{~cm}^{2}$; matrix size $(\mathrm{MTX})=128 \times 128$; slice thickness $(\mathrm{ST})=2 \mathrm{~mm}$; gap between slices $($ gap) $=0.5 \mathrm{~mm}$; number of slices $(\mathrm{NS})=25$-axial, 10 -sagittal, 11 -coronal; number of averages $(N A)=1$. High resolution T2-weighted fast spin-echo images were acquired afterwards in axial, sagittal and coronal planes containing the lesion and the contralateral side. Experimental parameters for these images were: $\mathrm{TEeff}=25 \mathrm{~ms} ; \mathrm{TR}=4 \mathrm{~s} ; \mathrm{ETL}=4 ; \mathrm{FOV}=6 \times 6 \mathrm{~cm}^{2}$; MTX $=512 \times 256-$ for axial and coronal sections and $256 \times 256$ for sagittal sections; $\mathrm{ST}=1 \mathrm{~mm}$; gap $=0.1 \mathrm{~mm}$; $\mathrm{NS}=35$-axial and coronal, and 32 -sagittal; NA=4 -axial and coronal, 2 -sagittal; experimental time $=12 \mathrm{~min} 48 \mathrm{~s}$ for axial and coronal images and $6 \mathrm{~min} 24 \mathrm{~s}$ for sagittal images. MRI data were acquired and processed on a Linux computer using Paravision 5.1 software (Bruker BioSpin GmbH, Ettlingen, Germany).

\section{Analysis of rat medial gastrocnemius muscle force}

The maximum tetanus force (TetF) of the gastrocnemius muscle was analyzed in rats $(\mathrm{N}=8)$ anesthetized by an intraperitoneal injection of a mixture of ketamine $(75 \mathrm{mg} / \mathrm{kg}$, i.p.) and xylazine 
$(10 \mathrm{mg} / \mathrm{kg}$, i.p.). Anesthetized animals were placed in prone position in a platform with the knee placed in fully extended position and fixed with 2 needles (one in the knee and the other in the ankle) in order to immobilize the limb. Achilles tendon including the calcaneus was separated from the surrounding tissue leaving the proximal origin, blood supply and nerves intact. The Achilles tendon was cut and attached, by using a tendon hook stretched with a passive force of $100 \mathrm{mN}$, to a force transducer (TRI-201 Letica Scientific Instruments, Barcelona, Spain) connected to a polygraph (Polygraph 2006; Letica Scientific Instruments, Barcelona, Spain). The sciatic nerve was then exposed through a lateral incision on the thigh and connected to an electrode and a stimulator (Universal Kimograph; Harvard Apparatus, Holliston, Massachusetts, USA). Muscle force analysis was conducted at a constant room temperature of $25-27^{\circ} \mathrm{C}$, and the gastrocnemius muscle was covered with mineral oil to prevent muscle drying during the muscle force determination. The TetF was measured by electrical stimulation of sciatic nerve at a frequency of $100 \mathrm{~Hz}, 0.1 \mathrm{~ms}$ pulse width and a voltage of $5 \mathrm{~V}$. For each animal, TetF was determined both in the control (noninjured) left gastrocnemius muscle and the injured right leg gastrocnemius muscle.

\section{Statistical analysis}

The statistical analysis was performed using IBM SPSS Statistics version 20.0 (IBM; Armonk, New York, United States). Maximum tetanus force values were normalized to the individual gastrocnemius muscle mass (represented by the TetF divided by the muscle weight and expressed in $\mathrm{mN} / \mathrm{g}$ ).

The normality and homoscedasticity of data were studied for each parameter to apply the correct statistical methods (parametric or non-parametric). The Kolmogorov-Smirnov test was used to verify the normal distribution of the data.

Data showed a normal distribution and paired Student's $t$ test were used to compare statistical differences in maximal muscle force between animal.

The power analysis for the t-paired test was automatically performed to detect a minimum difference in means of 0.8 and with and alpha value of 0.05 . Differences were considered statistically significant when $P<0.05$. The results are presented as mean \pm SD .

\section{Results}

$\boldsymbol{\nabla}$

\section{Analysis of surgically-induced muscle injury size in rat} medial gastrocnemius muscle

The size of the muscle injured area caused by the biopsy needle (० Fig. 1) was evaluated in $\mathrm{H}-\mathrm{E}$ stained muscle sections at $1,3,5$, $7,14,21,26,35$ and 46 days post-injury ( $\odot$ Fig. 2 ). Both injured muscle area and the total area of medial gastrocnemius muscle were quantified by using the Image J v1.46 software after marking out both the specific injured area and the total area of medial gastrocnemius muscle ( $\bullet$ Fig. $\mathbf{2 a}$ ). As shown in $\odot$ Fig. $\mathbf{2 b}$, the surgically-induced muscle injury with the biopsy needle caused a lesion in the rat medial gastrocnemius of $30.0 \pm 5.9 \%$ of the total transversal area of the muscle just $24 \mathrm{~h}$ post-injury, being at this time-point when the injured area reached its maximum size. From this time on, the measured size values of the injured area decreased progressively until the day 46 post-injury (at the end period of muscle regeneration process) when was approximately the $2 \%$ of the total area of medial gastrocnemius muscle.
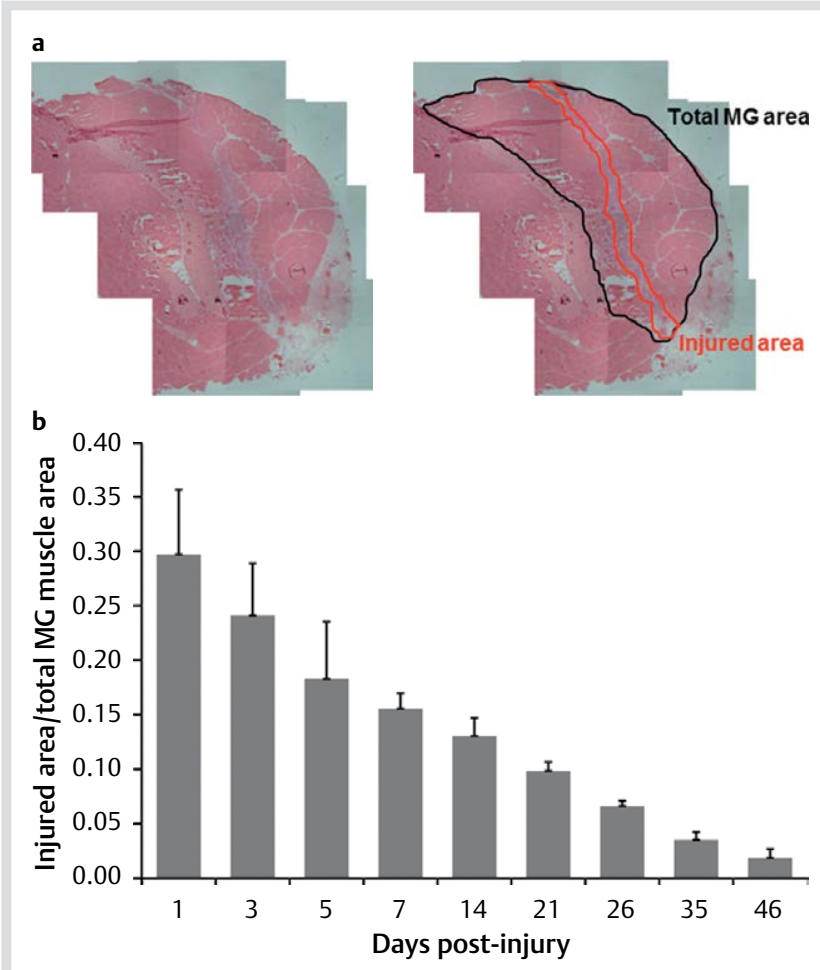

Fig. 2 Quantification of the size of the lesion induced by the biopsyneedle in the rat model. The size of the muscle injured area caused by the biopsy needle was evaluated in $\mathrm{H}$-E stained rat ( $\mathrm{N}=5$ for each time-point) muscle transversal sections at 1, 3, 5, 7, 14, 21, 26, 35 and 46 days post- injury. a Representative image (14 days post-injury) of the muscle $\mathrm{H}-\mathrm{E}$ stained sections used for measurement of the size of the injury. Both injured muscle area and the total area of medial gastrocnemius (MG) muscle were marked out and quantified by using the Image J v1.46 software. $\mathbf{b}$ The percentage of the injured muscle respecting to the total medial gastrocnemius muscle area was measured. Values are presented as the mean \pm SD of the injured area vs. total muscle area.

\section{Histological analysis of skeletal muscle injury longitudinal evolution}

By means of H-E stain ( $\odot$ Fig. 3), we corroborated that the histological changes in our rat model of skeletal muscle injury highly reproduced the necrosis and regeneration processes observed in other animal models of induced muscle injury $[11,26,39]$ or animal models of spontaneous skeletal muscle injury, such as the dystrophic mdx-mice [22]. As a result, 12-24h after surgicallyinduced injury our rat animal model showed the presence of necrotic fibers and edema with some infiltrating inflammatory cells and macrophages. This was followed by an inflammatory phase (1-3 days post-injury) were the injured area was extensively invaded by macrophages and inflammatory cells and an ensuing regenerative phase in which small diameter, newly formed, regenerating basophilic fibers started to appear (3-5 days post-injury). From 5-7 days post-injury, new formed basophilic fibers progressively grew in the injured area concomitantly with the progressive withdrawal of the inflammatory cells (7-14 days post-injury). 3 weeks after surgically-induced injury, muscle damaged tissue was completely replaced by the new muscle fibers. From 26 days after injury, the tissue showed a fully regenerated aspect with the presence of centrally-nuclei muscle fibers and the absence of inflammation-related cells. Finally, 46 days post-injury the tissue showed normal characteristics and completely regenerated structure. 


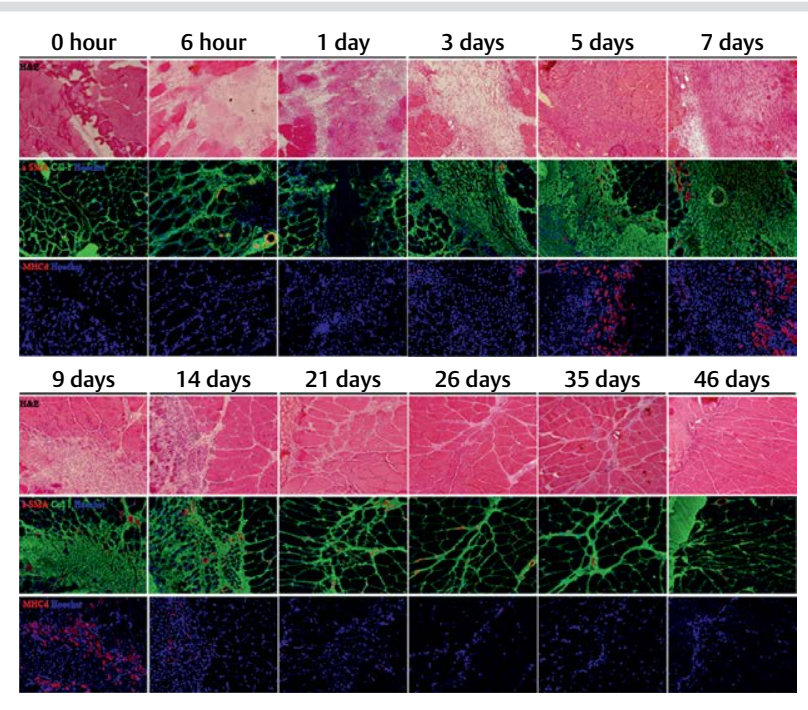

Fig. 3 Histologic and immunofluorescence analysis of the longitudinal evolution of skeletal muscle injury in the rat model. Representative images ( $\mathrm{N}=5$ for each time-point) show Hematoxylin-Eosin stained sections

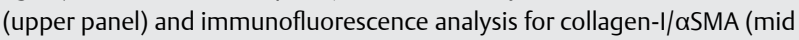
panel) and MHCd expression (lower panel) at different times ( 0 and $6 \mathrm{~h}$ and $1,3,5,7,9,14,21,26,35$ and 46 days post-injury) after the generation of surgically-induced medial gastrocnemius muscle injury in rats. Images show $(\times 100)$ microphotographs for all panels.

Immunofluorescence analysis of skeletal muscle injury longitudinal evolution

In order to determine the muscle regeneration and fibrogenesis experienced in our rat model of skeletal muscle injury, we performed a study by immunofluorescence ( $\odot$ Fig. 3 ) to analyze, on the one hand, the expression levels of the developmental/ embryonic form of myosin heavy chain (MHCd), a well-known marker for muscle development $[21,22]$ and adult muscle regeneration after injury [22] and, on the other hand, the levels of the fibrosis marker collagen-I and the angiogenesis marker $\alpha$-SMA, which stains for the smooth muscle layer of blood vessels and capillaries [14].

MHCd was analyzed during muscle necrosis and regeneration process by immunofluorescence. In adult muscle MHCd is only expressed during muscle regeneration after injury and in the first period of myofiber formation until its replacement by the mature isoforms (fast or slow) myosin heavy chains. In our skeletal muscle injury model, MHCd levels were undetectable during the first days post-injury. MHCd started to appear 3 days after the lesion and showed a progressive increase, reaching its maximum levels of expression around 7-9 days post-injury. From this time on, corresponding to the last phases of muscle regeneration and myofiber differentiation, MHCd expression decreased gradually to be undetectable beyond 26 days after muscle injury.

We also analyzed the expression levels of the fibrotic marker collagen-I during all the process of muscle regeneration after injury. Collagen-I, detected specifically surrounding the fibers' perimeter in non-injured muscle fibers, strongly increased its expression and deposition in injured areas just after the muscle lesion was created and persisted during the inflammatory and regeneration periods. Collagen-I showed a prompt and strong increase from the first days post-injury, demonstrating an abundant deposition in injured areas from day 3 post-injury and

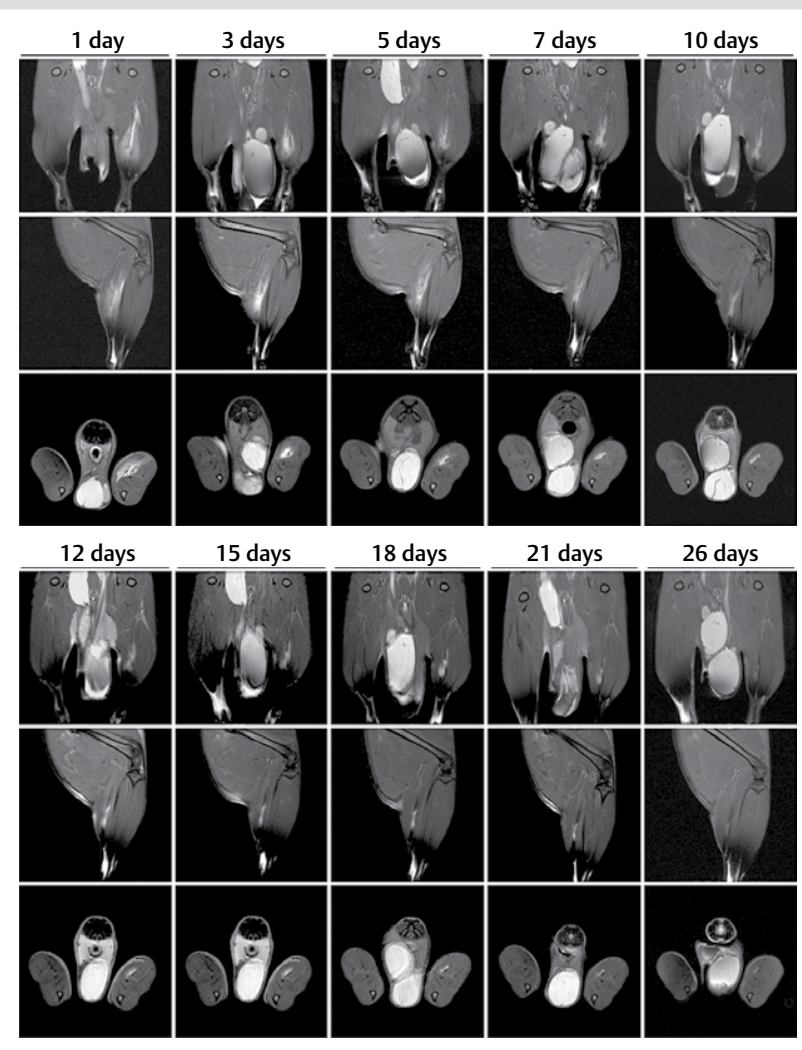

Fig. 4 In vivo MRI imaging analysis of the longitudinal evolution of skeletal muscle injury in the rat model. Representative images $(\mathrm{N}=3)$ show coronal (upper panel), sagittal (mid panel) and axial (lower panel) planes of the in vivo ${ }^{1} \mathrm{H}$-magnetic resonance imaging (MRI) studies performed at different times $(1,3,5,7,10,12,15,18,21$ and 26 days post-injury) after medial gastrocnemius muscle surgically-induced injury in rats. Both edema around central tendon and the typical high signal feather shape, which is characteristic of grade I-II muscle lesions in human athletes, was clearly visible from the first day post-injury. MRI studies were carried out by using a 7T Bruker BioSpec 70/30 USR (Bruker BioSpin GmbH, Ettlingen, Germany) system equipped with a mini-imaging gradient set $(400 \mathrm{mT} / \mathrm{m})$ and using a quadrature transceiver volume coil with $72 \mathrm{~mm}$ inner diameter. Experimental parameters for these images are indicated in Methods section. MRI data were acquired and processed on a Linux computer using Paravision 5.1 software (Bruker BioSpin GmbH, Ettlingen, Germany).

reaching its maximum levels around 7-9 days after injury. From day 14 post-injury, and coinciding with the presence of newly formed myofibers, collagen-I started to decrease progressively but its presence was still detectable 35 days after muscle lesion forming a scar-like tissue clearly visible following the direction were the lesion was created by crossing the muscle from side to side. The $\alpha$-SMA staining demonstrated the increase in the number of capillaries, specially in regenerating areas, from day 9 up to 21 post-injury where the new fibers are growing to regenerate the muscle tissue and the requirement of blood supply is increased ( $\bullet$ Fig. 3 ).

\section{MRI analysis of the longitudinal evolution of skeletal muscle injury}

We performed a longitudinal study of the lesion in the rat model by analyzing the evolution of skeletal muscle injury by using high resolution MRI either in the axial, coronal or sagittal planes. As shown in $\odot$ Fig. 4, the surgically-induced muscle injury produced a myotendinous break with retraction and gap. This mus- 
cle lesion caused a strong edema which was clearly detected at $24 \mathrm{~h}$ post-injury and localized around the central tendon. Remarkably, we observed the typical high signal feather shape, which is characteristic of the most frequently observed grade II-III muscle lesions in elite athletes. From this point on, edema progressively decreased until it was practically undetectable at 26 days post-injury.

\section{Surgically-induced skeletal muscle injury in rat model} mimics muscle lesions observed in human elite athletes Next, we evaluated the similarities of the surgically-induced lesion created in our rat model with those observed in professional athletes by comparing the MRI images obtained in rats with MRI images from elite soccer players. As shown in $\bullet$ Fig. 5, we demonstrated that our muscle injury model in rats highly reproduced the human lesions observed in professional athletes. In our rat model, the surgically-induced skeletal muscle injury caused a typical grade I-II lesion ( $\bullet$ Fig. 5a), which was highly comparable to the grade I and grade II lesions observed in the proximal long head biceps femoris of a 21 years-old and in the proximal head biceps of a 23 years-old male professional footballers, respectively ( $\bullet$ Fig. $\mathbf{5 b}, \mathbf{c}$ ). The muscle injury in the rat model caused an important edema and "feathery high signal" along skeletal muscle fibers which is typical of these types of injuries in professional athletes and highly mimicked the hyperintensity area of edema in form of feather representative of grade I lesions and the hypertensity area of edema, with gap and retraction were the tendon is commonly involved typical of grade II muscle injuries.

\section{Muscle force decrease after surgically-induced skeletal muscle injury}

The maximum tetanus force was measured 2 weeks after muscle injury both in control and in injured medial gastrocnemius muscles of each rat. As shown in 0 Fig. 6, injured muscles showed a significant decrease of the $29.1 \%(P=0.001)$ in the maximum contraction force demonstrating the efficacy of our new muscle injury approach in skeletal muscle force reduction. The mean values of TetF in control muscles and injured muscles were $643 \pm 163$ and $456 \pm 107 \mathrm{mN} / \mathrm{g}$, respectively.

\section{Discussion}

$\nabla$

In this study, we present a new animal model of surgicallyinduced skeletal muscle injury in rats, which is easy to reproduce and closely mimics the muscle lesions observed in human elite sportsmen. The new in vivo model of skeletal muscle injury will be helpful for researchers in the fields of skeletal muscle injuries and regeneration, as well as for the in vivo testing of new therapeutic approaches to prevent or ameliorate muscle lesions. By using our newly established technical approach of surgicallyinduced skeletal muscle injury, we were able not only to determine the size of the injury and all the histological and molecular changes in the muscle necrosis-regeneration process by histology and immunofluorescence but also to corroborate the similarities of our muscle injury rat model with the muscle lesions observed in professional athletes by using MRI imaging analysis. Furthermore, we evaluated the decrease in the medial gastrocnemius muscle force caused by the muscle injury in the rat model, in order to assess the impact on muscle strength related to surgically-induced lesions.
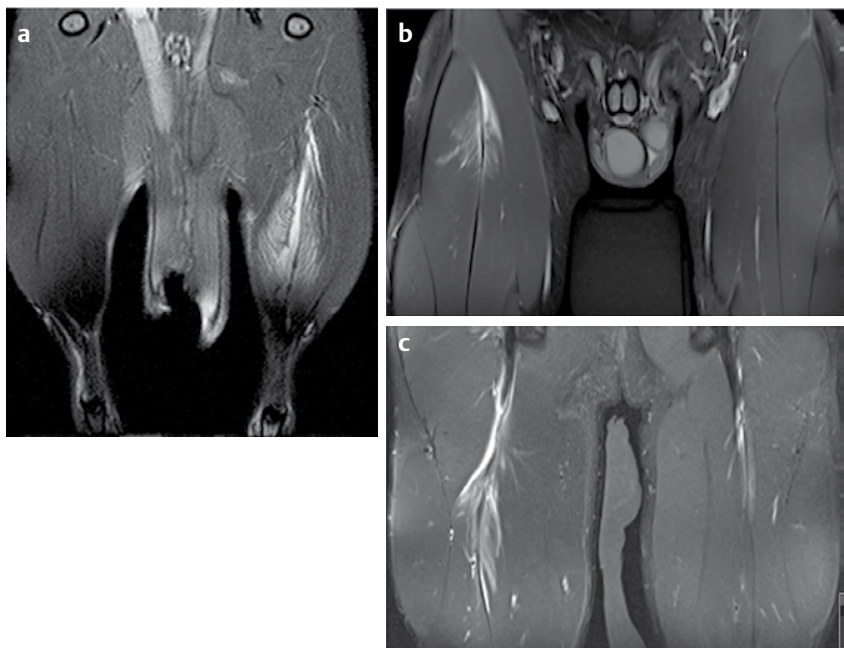

Fig. 5 MRI in vivo imaging of skeletal muscle lesion comparison between surgically-induced rat muscle injury model and human elite athletes. a Coronal T2 MRI image of a 2-month-old rat $24 \mathrm{~h}$ after injury in the medial gastrocnemius muscle. Image shows the edema and "feathery high signal" along the muscle fibers typical of these muscle injuries in human professional athletes. $\mathbf{b}$ Coronal T2 MRI image of the proximal rectus femoris of a 21-year-old male professional footballer showing a Grade I injury. Image shows small hyper intensity area of edema in feather-like shape. c Coronal T2 MRI image of the proximal head biceps showing a Grade II injury of a 23 year-old male professional footballer. Image shows important hypertensity area of edema, with gap and retraction and common tendon involved.

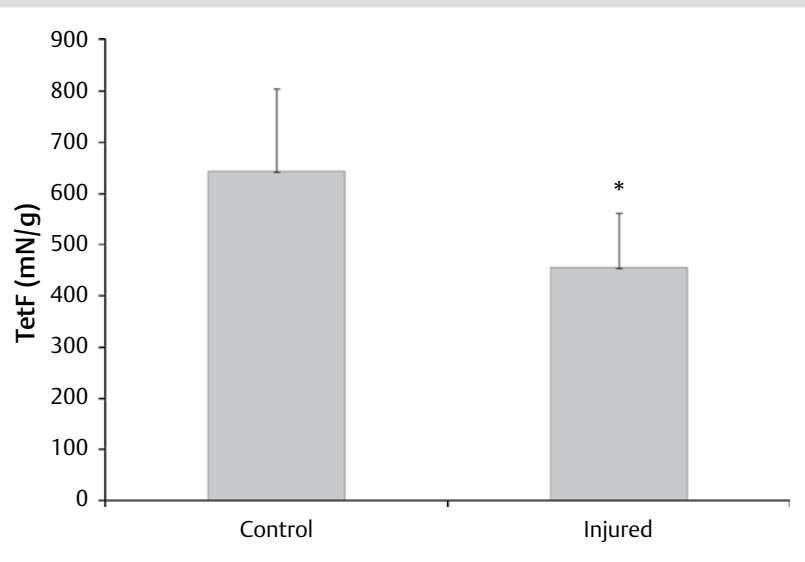

Fig. 6 Measurement of muscle strength in the surgically-induced muscle injury rat model. Maximum tetanus force (TetF) was measured in rats $(\mathrm{N}=8)$ after 2 weeks of surgically-induced muscle injury. TetF values were normalized to medial gastrocnemius muscle weight for the control and injured rat muscles. Values are presented as mean \pm SD $\left({ }^{*}\right.$ indicates $P=0.001)$.

The evolution of the muscle injury induced by the biopsy needle in the rat skeletal muscle was evaluated by H-E staining, immunofluorescence analysis of muscle regeneration and fibrosis markers, such as MHCd and Collagen-I, and MRI-based in vivo imaging analysis. By means of H-E stain ( $\odot$ Fig. 3), we corroborated that the histological changes in our rat model of skeletal muscle injury highly reproduced the necrosis, inflammation and regeneration processes observed in animal models of spontaneous skeletal muscle injury, such as the dystrophic mdx-mice [22]. We demonstrated that the surgically-induced muscle injury followed the same orchestrated process of skeletal muscle 
regeneration after injury. In this sense, we analyzed the expression of MHCd, a well-known marker of muscle regeneration and the formation of new fibers in adult muscle [22,29], which showed a similar pattern of expression than those detected in spontaneous muscle lesions in dystrophic animals [22]. MHCd expression increased from 3 to 14 days, indicating the presence of numerous newly formed regenerating fibers in the damaged area and then progressively disappears, becoming undetectable 21 days after injury coinciding with the maturation phase of regenerated fibers. On the other hand, we analyzed the possible intramuscular fibrosis induced in our model by determining the presence of the fibrotic marker type-I collagen. Type-I collagen expression showed a strong increase in the injured area from day 3 to day 9 after muscle damage. Beginning from day 14 postinjury type-I collagen deposition was progressively reduced, but a permanent scar-like type-I collagen positive extracellular matrix accumulation could be still detected one month after muscle lesion was created ( $\bullet$ Fig. 3). Muscle healing in sportsderived skeletal muscle injuries is commonly followed by scar tissue formation after injury [34]. Intramuscular scarring often represents an important sequel derived from muscle injuries in human athletes provoking muscle weakness and increasing the probability of muscle re-injury. Our skeletal muscle injurymodel therefore represents a useful approach to studying such measures as innovative therapeutic approaches to reduce the fibrous tissue accumulation or scarring prevention, by testing anti-fibrotic agents such as Suramin, a TGF- $\beta 1$ antagonist which has demonstrated its ability to reduce fibrous scar formation in muscle and enhance muscle strength in strain-injured skeletal muscle [5], or CAR-decorin, a potent inhibitor of scar formation by neutralization of both growth factors responsible for scarring, TGF- $\beta 1$ and $-\beta 2$ isoforms [20].

Since most of the human lesions related to sports are often evaluated by non-invasive imaging techniques such as MRI, we performed a longitudinal study in order to evaluate the progression of the disease and to compare our findings with the most common injuries detected in skeletal muscle of elite athletes. Remarkably, the comparison of the MRI analysis of the lesions caused by our surgical approach in rats showed a strong homology to grade I-II type lesions observed in elite soccer players ( $\bullet$ Fig. 5). Both edema and the feather-like shape was very similar between our rat model and elite soccer players after $24 \mathrm{~h}$ of skeletal muscle injury. As observed in human athletes, the surgically-induced muscle injury in rats showed a strong edema $24 \mathrm{~h}$ after muscle damage, which was slowly and progressively reabsorbed throughout the following weeks.

We also performed an analysis of the muscle force of rat skeletal muscle after injury, in order to determine the reduction in muscle strength after muscle injury in our rat model due to biopsy needle-induced muscle lesion. The measurement of the TetF demonstrated a decrease of approximately $30 \%$ of the total medial gastrocnemius muscle force 2 weeks after injury ( $\bullet$ Fig. 6 ) caused by the surgically-induced injury. Our results corroborate previous data reported in a toxin-induced muscle injury rat model, where a 37\% decrease in Tetanic force was described in rat gastrocnemius muscle 2 weeks after injury induced by intramuscular bupivacaine injection [24].

In summary, this study presents a new model of surgicallyinduced skeletal muscle injury in rats, which mimics the complete process of necrosis and regeneration observed in muscle injuries and reproduces very closely the most frequent skeletal muscle lesions observed in sports human clinics. This reproducible experimental animal model allows not only for the study of the complex regulatory pathways involved in the skeletal muscle degeneration-regeneration process but also opens new avenues for reliable assessment of the efficacy of both stem cell-based and pharmacological therapeutic approaches aimed at enhancing muscle tissue repair and preventing fibrosis and other adverse effects in sports-related skeletal muscle injuries.

\section{Acknowledgements}

$\nabla$

This study was supported by funds MTN20100101 and MTN2012005 from Fundación Leitat (Barcelona, Terrassa, Spain) and F. Futbol Club Barcelona (Barcelona, Spain) by its initiative MuscleTech Network (Barcelona, Spain). We thank Marta Rosal and Alejandro Rojo from the Animal Facility Unit of Vall d'Hebron Institut de Recerca (VHIR), Hospital Universitari Vall d'Hebron (Barcelona, Spain) and Silvia Lope from NMR service facilities (SeRMN) belonging to Universitat Autònoma de Barcelona (UAB, Bellaterra, Spain) for their valuable technical assistance.

Conflict of interest: The authors have no conflict of interest to declare.

\section{Affiliations}

${ }^{1}$ Leitat Foundation, Leitat Technological Center, Carrer de la Innovació 2, Terrassa, Barcelona, Spain

Bioengineering, Orthopedics and Pediatric Surgery Laboratory, Vall d'Hebron Institut de Recerca (VHIR), Universitat Autonoma de Barcelona, Barcelona, Spain

Physiology Department, Universitat de Barcelona, Barcelona, Spain ${ }^{4}$ Ultrasound Unit, Department of Radiology, Hospital Universitari Vall d'Hebron, Universitat Autònoma de Barcelona, Barcelona, Spain

School of Medicine, University of Tampere, Tampere, Finland

${ }^{6}$ Department of Orthopedics \& Traumatology, Tampere University Hospital, Tampere, Finland

${ }^{7}$ Translational Research in Fetal Surgery for Congenital Malformations Laboratory, Center for Fetal, Cellular and Molecular Therapy, Cincinnati Children's Hospital Medical Center (CCHMC), Cincinnati, Ohio, USA ${ }^{8}$ Medical Services, Futbol Club Barcelona, Ciutat Esportiva Futbol Club Barcelona, Barcelona, Spain

\section{References}

1 Aspelin P, Ekberg O, Thorsson O, Wilhelmsson M, Westlin N. Ultrasound examination of soft tissue injury of the lower limb in athletes. Am J Sports Med 1992; 20: 601-603

2 Best TM, Hunter KD. Muscle injury and repair. Phys Med Rehabil Clin N Am 2000; 11: 251-266

3 Bischoff R. In: Engel AG, Franszini-Armstrong C (eds.). The satellite cell and muscle regeneration. In Myogenesis. New York: McGrawHil; 1994: 97-118

4 Bubnov R, Yevseenko V, Semeniv I. Ultrasound guided injections of platelets rich plasma for muscle injury in professional athletes. Comparative study. Med Ultrason 2013; 15: 101-105

5 Chan Y-S, Li Y, Foster W, Fu FH, Huard J. The use of suramin, an antifibrotic agent, to improve muscle recovery after strain injury. Am J Sports Med 2005; 33: 43-51

6 Chargé SBP, Rudnicki MA. Cellular and molecular regulation of muscle regeneration. Physiol Rev 2004; 84: 209-238

7 Ebbeling CB, Clarkson PM. Exercise-induced muscle damage and adaptation. Sports Med 1989; 7: 207-234

8 Goetsch SC, Hawke TJ, Gallardo TD, Richardson JA, Garry DJ. Transcriptional profiling and regulation of the extracellular matrix during muscle regeneration. Physiol Genomics 2003; 14: 261-271

9 Grounds MD, Yablonka-Reuveni Z. Molecular and cell biology of skeletal muscle regeneration. Mol Cell Biol Hum Dis Ser 1993; 3: 210-256

10 Hamilton B, Valle X, Rodas G, Til L, Pruna Grive R, Gutierrez Rincon Ja, Tol JL. Classification and grading of muscle injuries: a narrative review. Br J Sports Med 2014; 49: 306 
11 Harris JB, Johnson MA. Further observations on the pathological responses of rat skeletal muscle to toxins isolated from the venom of the Australian tiger snake, Notechis scutatus scutatus. Clin Exp Pharmacol Physiol 1978; 5: 587-600

12 Harriss DJ, Atkinson G. Ethical standards in sport and exercise science research: 2014 update. Int J Sports Med 2013; 34: 1025-1028

13 Hawke TJ, Garry DJ. Myogenic satellite cells: physiology to molecular biology. J Appl Physiol 2001; 91: 534-551

14 Horio T, Fujita M, Tanaka Y, Ishihara M, Kishimoto S, Nakamura S, Hase $K$, Maehara T. Efficacy of fragmin/protamine microparticles containing fibroblast growth factor-2 (F/P MPs/FGF-2) to induce collateral vessels in a rabbit model of hindlimb ischemia. J Vasc Surg 2011; 54: 791-798

15 Huard J, Li Y, Fu FH. Muscle injuries and repair: current trends in research. J Bone Joint Surg Am 2002; 84-A: 822-832

16 Hurme T, Kalimo H, Lehto M, Järvinen M. Healing of skeletal muscle injury: an ultrastructural and immunohistochemical study. Med Sci Sports Exerc 1991; 23: 801-810

17 Järvinen TA, Järvinen $M$, Kalimo $H$. Regeneration of injured skeletal muscle after the injury. Muscles Ligaments Tendons J 2013; 3: 337345

18 Järvinen $T A H$, Järvinen $T L N$, Kääriäinen $M$, Aärimaa $V$, Vaittinen $S$, Kalimo H, Järvinen $M$. Muscle injuries: optimising recovery. Best Pract Res Clin Rheumatol 2007; 21: 317-331

19 Järvinen TAH,Järvinen TLN, Kääriäinen M, Kalimo H,Järvinen M. Muscle injuries: biology and treatment. Am J Sports Med 2005; 33: 745-764

20 Järvinen TAH, Ruoslahti E. Target-seeking antifibrotic compound enhances wound healing and suppresses scar formation in mice. Proc Natl Acad Sci USA 2010; 107: 21671-21676

21 Lehto MU, Järvinen MJ. Muscle injuries, their healing process and treatment. Ann Chir Gynaecol 1991; 80: 102-108

22 Marotta M, Sarria Y, Ruiz-Roig C, Munell F, Roig-Quilis M. Laser microdissection-based expression analysis of key genes involved in muscle regeneration in mdx mice. Neuromuscul Disord 2007; 17: 707-718

23 Mauch F, Best R, Bauer G. Current treatment concepts for muscular injuries. Unfallchirurg 2013; 116: 488-496

24 McNeill Ingham SJ, de Pochini AC, de Oliveira DA, Garcia Lisboa BC, Beutel A, Valero-Lapchik VB, Ferreira AM, Abdalla RJ, Cohen M, Han SW. Bupivacaine Injection leads to muscle force reduction and histologic changes in a murine model. PM\&R 2011; 3: 1106-1109

25 Meeson AP, Hawke TJ, Graham S, Jiang N, Elterman J, Hutcheson K, Dimaio JM, Gallardo TD, Garry DJ. Cellular and molecular regulation of skeletal muscle side population cells. Stem Cells 2004; 22: 1305-1320
26 Mitchell CA, McGeachie JK, Grounds MD. Cellular differences in the regeneration of murine skeletal muscle: A quantitative histological study in SJL/J and BALB/c mice. Cell Tissue Res 1992; 269: 159-166

27 Pollock N, James SLJ, Lee JC, Chakraverty R. British athletics muscle injury classification: a new grading system. Br J Sports Med 2014; 48: 1347-1351

28 Rantanen J, Hurme T, Lukka R, Heino J, Kalimo H. Satellite cell proliferation and the expression of myogenin and desmin in regenerating skeletal muscle: evidence for two different populations of satellite cells. Lab Invest 1995; 72: 341-347

29 Roig-Quilis M, Roma J, Marotta M, Sarria Y, Fargas A. Muscle regeneration following glycerol injection mimic that of mdx-mice degenerative-regenerative groups. Rev Neurol 2004; 38: 1101-1108

30 Scholz D, Thomas S, Sass S, Podzuweit T. Angiogenesis and myogenesis as two facets of inflammatory post-ischemic tissue regeneration. Mol Cell Biochem 2003; 246: 57-67

31 Schultz E, McCormick KM. Skeletal muscle satellite cells. Rev Physiol Biochem Pharmacol 1994; 123: 213-257

32 De Smet AA, Best TM. MR imaging of the distribution and location of acute hamstring injuries in athletes. Am J Roentgenol 2000; 174: 393-399

33 Timpka T, Jacobsson J, Bickenbach J, Finch CF, Ekberg J, Nordenfelt L. What is a sports injury? Sports Med 2014; 44: 423-428

34 Vaittinen S, Hurme T, Rantanen J, Kalimo H. Transected myofibres may remain permanently divided in two parts. Neuromuscul Disord 2002; 12: $584-587$

35 Wagatsuma A. Endogenous expression of angiogenesis-related factors in response to muscle injury. Mol Cell Biochem 2007; 298: 151-159

36 Whalen RG, Harris JB, Butler-Browne GS, Sesodia S. Expression of myosin isoforms during notexin-induced regeneration of rat soleus muscles. Dev Biol 1990; 141: 24-40

37 Woods C, Hawkins RD, Maltby S, Hulse M, Thomas A, Hodson A. The Football Association Medical Research Programme: an audit of injuries in professional football - analysis of hamstring injuries. $\mathrm{Br} \mathrm{J}$ Sports Med 2004; 38: 36-41

38 Woolf $A D$, Pfleger $B$. Burden of major musculoskeletal conditions. Bull World Health Organ 2003; 81: 646-656

39 Yan Z, Choi S, Liu X, Zhang M, Schageman JJ, Lee SY, Hart R, Lin L, Thurmond FA, Williams RS. Highly coordinated gene regulation in mouse skeletal muscle regeneration. J Biol Chem 2003; 278: 8826-8836 